\title{
CONTEXTO Y EVOLUCIÓN DEL PLAN DE MANEJO INTEGRAL DE RESIDUOS SÓLIDOS EN LA UNIVERSIDAD IBEROAMERICANA CIUDAD DE MÉXICO
}

\author{
Mariana RUIZ MORALES
}

Departamento de Ingenierías, Universidad Iberoamericana Ciudad de México, Prolongación Paseo de la Reforma 880, Lomas de Santa Fe, Ciudad de México 01219, México. Correo electrónico: mariana.ruiz@ibero.mx

(Recibido febrero 2016; aceptado septiembre 2016)

Palabras clave: caracterización, reducción, separación de residuos, reciclaje, gestión ambiental

\section{RESUMEN}

El presente artículo muestra los resultados de la implementación de un Plan de Manejo Integral de Residuos Sólidos en la Universidad Iberoamericana Ciudad de México cuyo objetivo ha sido reducir la cantidad de residuos que se generan en la institución así como aumentar la tasa de separación de residuos reciclables y el composteo. Las acciones llevadas a cabo durante el período 2009-2015 incluyen la puesta en marcha de diversas políticas además de la instalación de un sistema de contenedores para residuos reciclables de tipo modular para facilitar su separación. Los resultados muestran una disminución de casi un $24 \%$ en la generación total de residuos y un incremento promedio de $144 \%$ en la separación de los residuos reciclables en el período estudiado. El Plan Integral no se limita a la responsabilidad de la administración universitaria sino que también se orienta a ser una herramienta educativa para la cultura ambiental fomentando la participación de los alumnos en los proyectos relacionados con la cuantificación, caracterización y manejo integral de los residuos.

Key words: characterization, reduction, waste sorting, recycling, waste management

\begin{abstract}
This paper shows the results of the establishment of an Integrated Solid Waste Management Program at Universidad Iberoamericana Mexico City, aimed to reduce the amount of waste generated and to increase the rates of recycling and composting. Some of the actions that have been implemented in the period from 2009 to 2015 include the application of several policies and the employment of a modular container system for recyclable materials. Results show a $24 \%$ decrease in total waste generation and a $144 \%$ increase in the recovery rate of recyclable materials in the analyzed period. The Program is not limited to the responsibility of campus administration, but it is also intended to be an educational tool for environmental awareness, promoting student participation in projects of waste quantification, characterization, and management.
\end{abstract}




\section{INTRODUCCIÓN}

La protección del ambiente está siendo cada vez más reconocida como un factor clave en la salud pública. Las instituciones de educación superior, en su papel no sólo de investigación y docencia sino también como espacios de convivencia y formación de hábitos y actitudes, han emprendido diversas acciones en busca de la sustentabilidad en los ámbitos de energía, movilidad, agua y manejo de residuos sólidos. Dichas acciones no sólo tienen un impacto en la gestión ambiental de la institución sino también un impacto educativo, ya que lo que ven y viven los estudiantes sobre las prácticas ambientales impacta en su formación, es decir, que la gestión ambiental trasciende de un asunto meramente administrativo en las universidades (Gutiérrez Barba y Martínez Rodríguez 2010).

Es por ello que, en años recientes, las iniciativas de diversas universidades en México se han unido para formar el Consorcio Mexicano de Programas Ambientales Universitarios para el Desarrollo Sustentable, en el cual se busca la articulación de las funciones sustantivas de las instituciones de educación superior (docencia, investigación y extensión) con las funciones adjetivas (administración y apoyo) en torno al ambiente y al desarrollo sustentable (Nieto-Caraveo 2001). Se busca construir un proceso educativo integral que, entre otras cosas, favorezca la disminución del deterioro ambiental y la conservación de la diversidad biológica y cultural (Martín 2005). De igual forma, la Asociación Nacional de Universidades e Instituciones de Educación Superior presenta en su Plan de Acción para el Desarrollo Sustentable una visión al 2020 donde las instituciones incorporan la dimensión ambiental en los planes de desarrollo institucional, incluyendo acciones de transmisión, generación, aplicación y difusión (Gutiérrez Barba y Martínez Rodríguez 2010). En concordancia con lo anterior, la Universidad Iberoamericana Ciudad de México aprobó en 2016 la Política Institucional de Sustentabilidad, con la cual la institución refrenda su compromiso con la realidad social y ambiental de México. Esta Política consta de 13 principios que orientan la incorporación transversal de la perspectiva de la sustentabilidad en las funciones de docencia, investigación, vinculación y gestión del campus (Fernández Dávalos 2016).

El presente estudio se centra de forma particular en el tema de residuos sólidos ya que, debido a las grandes cantidades de residuos generados en las universidades, resulta indispensable buscar alternativas para su reducción y su separación a fin de lograr un reciclaje eficiente. A la vez, el tema de residuos representa una oportunidad de educación ambiental práctica, al promover la participación colaborativa de toda la comunidad universitaria. En este trabajo se incluye una revisión de la visión internacional y nacional sobre lo que las instituciones de educación superior han implementado referente al manejo de residuos y se detalla lo realizado por la Universidad Iberoamericana Ciudad de México. Se espera que esta experiencia sea un aporte que ayude a otras universidades a implementar estrategias similares.

\section{Visión internacional de las instituciones de edu- cación superior en planes de manejo de residuos sólidos}

La literatura reporta casos de gestión de residuos sólidos en universidades de diversos países del mundo. En China, la Universidad Shenyang ha implementado un modelo de universidad verde con un sistema integral de gestión de residuos sólidos que es ejemplo para otras universidades chinas (Geng et al. 2013). En Nueva Zelanda hay estudios sobre los comportamientos de los estudiantes frente a la separación de residuos reciclables (Long et al. 2014).

Tras una revisión realizada de las diversas páginas web de universidades norteamericanas, las que presentan los programas más notables de separación de residuos reciclables son: Universidad de Stanford, Universidad de Nueva York campus Washington Square, Universidad de Harvard, Universidad Estatal de California en San Marcos, Universidad Estatal de Carolina del Norte, Universidad de Oregon y Universidad de Cornell donde se promueve el principio R5: Respetar, Repensar, Reducir, Reusar y Reciclar. En ese país, la oportunidad de intervención en el tema de separación de residuos reciclables en las universidades es relevante dada la cantidad de residuos sólidos que se genera y la infraestructura con que cuenta la mayoría de las instituciones de educación superior (Largo-Wight et al. 2013). Posey y Webster (2013) analizaron las prácticas sustentables de las instituciones de educación superior técnica en el sur de los Estados Unidos. Su estudio resalta que los temas de mayor presencia son: conservación de energía, separación de residuos sólidos, reducción de residuos, construcción y renovación de edificios y reducción del uso de materiales tóxicos. Otros resultados muestran que las políticas en tema de residuos son coincidentes en diversas universidades, en la mayoría de los casos la principal estrategia de reducción en la generación de residuos estriba en la utilización del papel por ambos lados y la principal estrategia de reúso se centra en la donación de muebles y equipos de cómputo (Blumenstein 2008, Goral 2009, Atherton 
y Giurco 2011). En cuanto al reciclaje de residuos orgánicos, $75 \%$ de las principales universidades de Estados Unidos recicla los residuos de jardinería a través del composteo y $45 \%$ lo hace con los residuos de alimentos (Fournier 2008).

En el caso de Latinoamérica, se llevó a cabo un estudio con la participación de 65 universidades localizadas en 10 países (ARIUSA 2014). Dicho estudio mostró que las dimensiones en las que las universidades aparecen con resultados más favorables son la responsabilidad socioambiental, las políticas de sustentabilidad y la gestión de residuos. De hecho, el estudio indica que el manejo de residuos es el aspecto que más está ocupando la atención de las universidades. Se observa que $26 \%$ de las universidades estudiadas realizan procesos de minimización, separación y manejo adecuado de los residuos orgánicos provenientes de cafeterías, residencias universitarias y podas de jardines, mientras que $51 \%$ cuentan con un sistema de información y seguimiento de residuos sólidos por tipo y cantidad. El análisis también sugiere que a más antigüedad, dispersión de campus y mayor número de carreras experimentales, mayor es el desarrollo de políticas de sustentabilidad en las universidades. Según esta misma publicación, siempre resulta más fácil y económico promover programas de sensibilización que medidas de gestión, aunque la efectividad real de las medidas de sensibilización ambiental suele ser baja.

\section{Experiencias a nivel nacional}

En México, los estudios más relevantes son los llevados a cabo por la Universidad Nacional Autónoma de México (Alcántara et al. 2005), la Universidad Autónoma Metropolitana (Espinosa Valdemar et al. 2013), la Universidad Autónoma de Baja California (Armijo et al. 2008) y la Universidad Iberoamericana Ciudad de México (Ruiz Morales 2012). Estas publicaciones coinciden en que los residuos generados por instituciones educativas contienen un alto porcentaje de residuos reciclables (superior al $60 \%$ ). El estudio llevado a cabo por Espinosa Valdemar et al. (2013) en la Universidad Autónoma Metropolitana Azcapotzalco (con una superficie de 20 ha - 13 construidas y población de 15000 alumnos y 2800 trabajadores) revela que gracias al Programa de Gestión Integral de Residuos Sólidos, se han enviado a reciclaje más de 77 toneladas de residuos entre 2003 y 2011.

La principal limitante es que aún existen muchas instituciones de educación superior que desconocen con certeza cuántos residuos sólidos generan, siendo que la mayoría de las publicaciones con respecto a este tema en México se centran en la caracterización de residuos comerciales (Fierro Ochoa et al. 2010, Garduño Palomino 2012) y en el análisis de generación per cápita de la población en general (Saldaña et al. 2013, Taboada- González et al. 2013).

\section{DESCRIPCIÓN DEL PLAN DE MANEJO INTEGRAL DE RESIDUOS SÓLIDOS EN LA UNIVERSIDAD IBEROAMERICANA CIUDAD DE MÉXICO}

La Universidad Iberoamericana Ciudad de México tiene una superficie de $193352 \mathrm{~m}^{2}$ y una población de 13953 (datos de 2014) contando alumnos de licenciatura, posgrado, nivel técnico y preparatoria, personal académico, administrativo y de servicio y se ubica en el grupo de grandes generadores de residuos sólidos (más de 10 t/año).

Si bien desde el año 2000 se ha promovido la separación de los residuos reciclables, fue a raíz del proyecto Ibero Campus Verde que en el año 2009 se realizó el primer estudio de cuantificación y caracterización de residuos que motivó la puesta en marcha de un Plan de Manejo Integral de Residuos Sólidos. Este plan ha tenido por objetivo reducir la cantidad de residuos que se generan en la institución, así como aprovechar o enviar a reciclaje la mayor cantidad posible de los residuos generados y dar un manejo ambientalmente adecuado a aquellos que se entregan a los servicios municipales de limpia. Para el año 2013 Ibero Campus Verde se planteó una serie de objetivos cubriendo diversas áreas en temas de comunicación y formación, operación y gestión responsable de residuos según se ejemplifica en la figura 1.

El Plan de Manejo Integral de Residuos Sólidos incluye una serie de acciones de diversa índole y objetivo que se implementaron en la Universidad a partir del año 2008. Estas acciones se pueden englobar en las categorías siguientes: a) reducción, b) manejo y separación y c) educación.

a) Reducción: Para la reducción en el consumo y la generación de botellas de polietileno (PET), se instaló una planta purificadora de agua que abastece los garrafones en el campus a fin de evitar la compra de agua embotellada para las oficinas. Adicionalmente, se instalaron bebederos y estaciones para rellenar botellas con agua potable disponibles para toda la comunidad y particularmente en lugares estratégicos para los alumnos, siendo ellos la mayor parte de la población universitaria (aproximadamente el $70 \%$ ). En el caso del papel, se inició el cobro a los alumnos de las impresiones realizadas en los centros de 


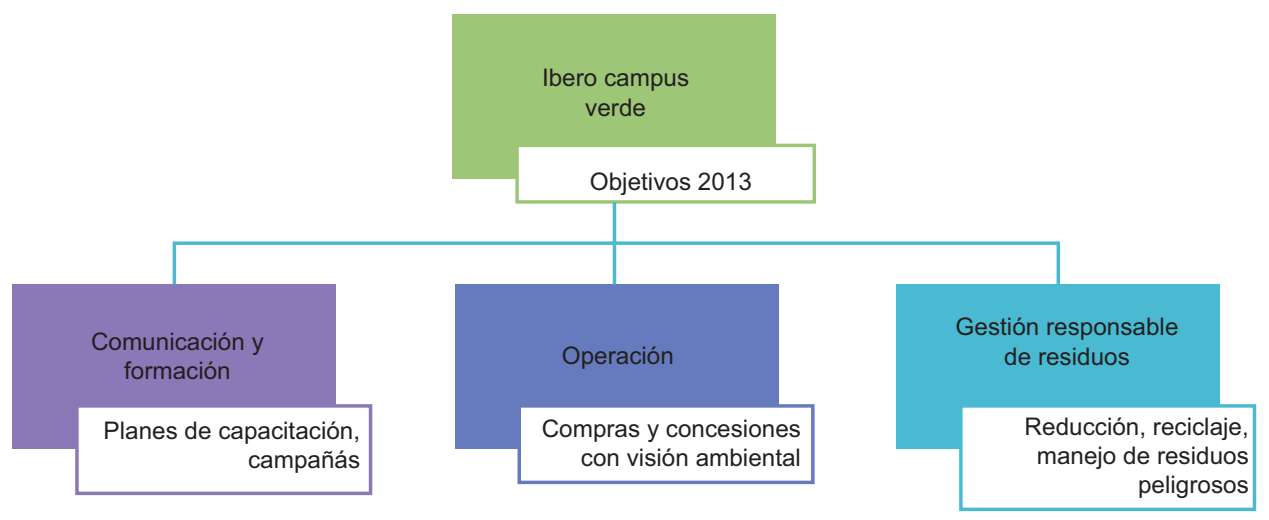

Fig. 1. Objetivos para 2013 del proyecto Ibero Campus Verde, elaborado con información de IBERO CAMPUS VERDE 2012

cómputo de la Universidad, disminuyendo el abuso en las impresiones de documentos innecesarios. Por otro lado, en las diversas oficinas se lleva a cabo una sustitución progresiva de tecnología en las impresoras y fotocopiadoras con un control con credencial que asegura que los usuarios recojan los documentos que envían a impresión y se lleve un conteo de las impresiones y fotocopias que se obtienen. Además, diversas áreas de la Universidad modificaron sus procesos para reducir el consumo de papel, como por ejemplo, con la distribución electrónica de boletines.

b) Manejo y separación. Las cafeterías ubicadas dentro de la Universidad separan los residuos orgánicos e inorgánicos, además del aceite utilizado para cocinar que es entregado a una empresa especializada para convertirlo en combustible. Desde 2008 la Universidad cuenta con una estación experimental de lombricomposta a cargo del Departamento de Ingenierías, la cual procesa una fracción de los residuos provenientes de las cafeterías. En cuanto a los residuos de jardinería, estos son enviados a la Planta de Composteo de la Delegación Álvaro Obregón.

En lo referente a residuos inorgánicos, a mediados de 2014 se renovaron los contenedores para disponer de residuos reciclables, instalándose 80 módulos de separación en los sitios de mayor circulación. Estos módulos están elaborados a partir de plástico reciclado y fueron diseñados primordialmente para la separación de latas de aluminio, papel y botellas de PET. Cuatro de estos módulos también están habilitados para separar vidrio y empaques de Tetra Pak ${ }^{\circledR}$. De igual manera, se instalaron cuatro contenedores para la recolección de cartuchos de tóner y tinta, teléfonos celulares y pilas. Se informó a la comunidad universitaria sobre la instalación de estos contenedores pero no se realizó ninguna campaña masiva en particular, ya que se de- seaba averiguar si la instalación de los nuevos módulos bastaba para incrementar la separación de los residuos, puesto que el objetivo de los nuevos módulos era hacer la separación de residuos reciclables más sencilla y conveniente para el usuario. Para ello, se optó por una alimentación frontal en lugar de vertical a través de una abertura de tamaño y forma distintivos para cada material y la colocación de señalización informativa (Fig. 2). Con esta acción, la Universidad se ha planteado como meta separar al menos 50 por ciento de sus residuos reciclables. Por otro lado, desde el año 2013 se cuenta con un programa para el acopio de bolsas aluminizadas de botanas y galletas que, a través de la iniciativa Terracycle ${ }^{\circledR}$, son enviadas a reciclaje. Este programa se realiza con la participación voluntaria de profesores y alumnos, quienes pueden traer este tipo de empaques de sus hogares u oficinas y en ningún momento se fomenta el consumo de algún producto en particular. En un lapso de tres años se han enviado a reciclaje más de 45000 empaques.

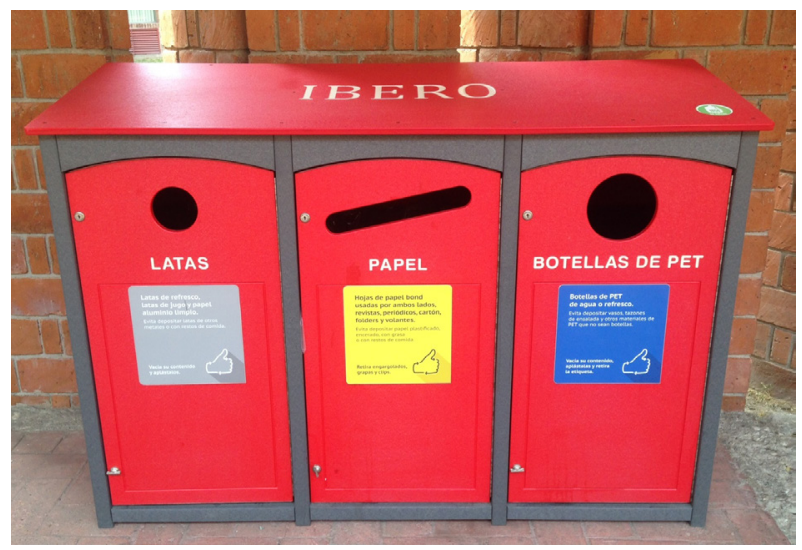

Fig.2. Módulo de separación de residuos reciclables de la Universidad Iberoamericana Ciudad de México 
Para los residuos electrónicos, semestralmente se lleva a cabo el evento "Electrorecicla", durante el cual, los miembros de la comunidad pueden traer de sus casas los equipos que desean desechar y junto con los residuos electrónicos generados en la Universidad se entregan a una compañía especializada para su correcta disposición. Finalmente, el mobiliario (mesas, archiveros, libreros, etc.) y equipo en buen estado que ya no se utiliza se dona a escuelas públicas y a obras sociales.

c) Educación. Desde el año 2000 el Programa de Medio Ambiente ha promovido iniciativas de discusión interdisciplinaria que han dado origen a programas de educación continua, seminarios de sustentabilidad y la creación de una política de sustentabilidad. En dichos grupos de trabajo participan áreas diversas de la academia y gestión universitaria y se cuestiona el modelo de desarrollo actual que origina en gran medida la problemática de residuos sólidos. Por otro lado, en el periodo 2009-2012 se llevaron a cabo las siguientes campañas: "Reduce tu huella", orientada a promover el cambio de hábitos con el fin de reducir la huella ecológica; "Cambia tu PET", con el propósito de promover el uso de envases reusables entregando, a través de un patrocinio, cerca de 5000 envases para bebidas; “¿Cuál es tu papel?”, que convocó a la comunidad a identificar procesos o trámites que tuvieran un excesivo consumo de papel y a proponer una alternativa viable de reducción; "Reduce, Reúsa, Recicla" a través de la cual se colocaron señalamientos en los contenedores de separación de residuos reciclables y se montaron figuras humanas elaboradas con PET para llamar la atención de la comunidad hacia los contendores y, finalmente, "Haz un buen papel", orientada a sensibilizar a la comunidad universitaria sobre el impacto ambiental de la fabricación del papel y promover su uso eficiente entregándose a todos los profesores dispositivos de almacenamiento USB que contenían un video referente al tema, además de recomendaciones para reducir el consumo de papel en la impartición de clases.

A la fecha se han realizado tres estudios de cuantificación y caracterización de los residuos sólidos urbanos de la Universidad, todos en el mes de abril de los años 2009, 2012 y 2015. Estos estudios han permitido determinar la cantidad total de residuos sólidos generados diariamente y la cantidad que se genera de cada subproducto. La más reciente cuantificación y caracterización de residuos se realizó, como en años anteriores, conforme a la Norma Oficial Mexicana NMX-AA-015-1985 (SECOFI 1985a), NMXAA-022-1985 (SECOFI 1985b) y NMX-AA-61-1985 (SECOFI 1985c), a partir de un muestreo aleatorio en el mes de abril de 2015. El estudio tuvo la participación de 16 alumnos de la licenciatura en Ingeniería Industrial. Se llevó a cabo la cuantificación mediante el pesaje total de los residuos generados utilizando una báscula mecánica industrial con capacidad de $500 \mathrm{~kg}$ y una balanza electrónica con capacidad de $50 \mathrm{~kg}$ y sensibilidad de $10 \mathrm{~g}$. Para la caracterización se utilizó el método de cuarteo. Se tomaron nueve bolsas de desechos con un peso total de $270 \mathrm{~kg}$ (muestra de cuarteo). Los desechos se vaciaron sobre una superficie de $4 \times 4 \mathrm{~m}$ y se homogeneizaron los residuos revolviéndolos con pala. Se dividió en cuatro partes aproximadamente iguales y se eliminaron las partes opuestas en diagonal. El restante se volvió a revolver hasta homogeneizar y se repitió la división dos veces más hasta obtener un aproximado de $33 \mathrm{~kg}$ (muestra para caracterización) para la selección de subproductos. Se estima la exactitud de este estudio en $+/-10 \%$ debido a errores humanos.

Los componentes obtenidos de la muestra para caracterización fueron agrupados en categorías según se muestra en la sección de resultados. Esta información fue comparada con los estudios previos (2009 y 2012) al igual que los datos recopilados sobre residuos separados y enviados a reciclaje en dichos períodos.

\section{RESULTADOS DE LAS ACCIONES DEL PLAN DE MANEJO}

A fin de determinar la efectividad de las diversas acciones que conforman el Plan de Manejo Integral de Residuos Sólidos, se analizaron primordialmente:

- Los resultados de los estudios de cuantificación y caracterización de residuos generados, a fin de determinar en qué grado se logra el objetivo de la reducción de residuos.

- Los datos de porcentajes de residuos que se logaron separar para ser enviados a reciclaje, pues es ahí donde queda de manifiesto si se logran los objetivos relacionados con el adecuado manejo de los residuos.

- La comparación de los datos anteriores en los periodos 2009, 2012 y 2015 a fin de terminar los posibles efectos de la evolución del Plan.

En cuanto a la cuantificación de residuos sólidos, se obtuvo que la generación típica máxima en la Universidad pasó de 3.375 t/día en 2009 a 2.712 t/día en 2012, es decir, una reducción de casi $20 \%$ y en 2015 a 2.571 Ton/día, lo que representa una 
reducción de $5 \%$ adicional, o una reducción total de alrededor de $25 \%$ en el período 2009-2015. Este resultado puede ser un indicador de la efectividad de las diversas acciones realizadas para la reducción de residuos que se indican en la sección "Descripción del Plan de Manejo Integral de Residuos Sólidos en la Universidad Iberoamericana Ciudad de México". También es importante analizar los resultados por tipo de residuos. Al comparar la caracterización de los residuos de 2015 con las caracterizaciones anteriores (2009 y 2012), se observa que se redujo la generación de algunos residuos mientras que de otros se incrementó notablemente. En la figura 3 se muestra un comparativo de la generación de cada tipo de residuo en los tres diferentes períodos en que se llevó a cabo el estudio. Como puede observarse en esta gráfica, los cambios más notorios en el período de los seis años son la diminución de residuos alimenticios y residuos de papel y el aumento en los residuos sanitarios y de plástico mixto, aunque cabe señalar que en el análisis detallado del plástico, se observó una reducción en la generación de PET. La reducción en la generación de los residuos reciclables se puede ver en el cuadro I. Se observa que durante los primeros tres años del programa todos los residuos reciclables tuvieron una reducción en la generación que va desde el $16 \%$ hasta el $65 \%$, mientras que al contabilizar 6 años del programa la reducción va del $19 \%$ al $69 \%$.

Con respecto a los resultados de la separación de residuos, éstos pueden observarse en el cuadro II en porcentaje de separación por tipo de residuo para

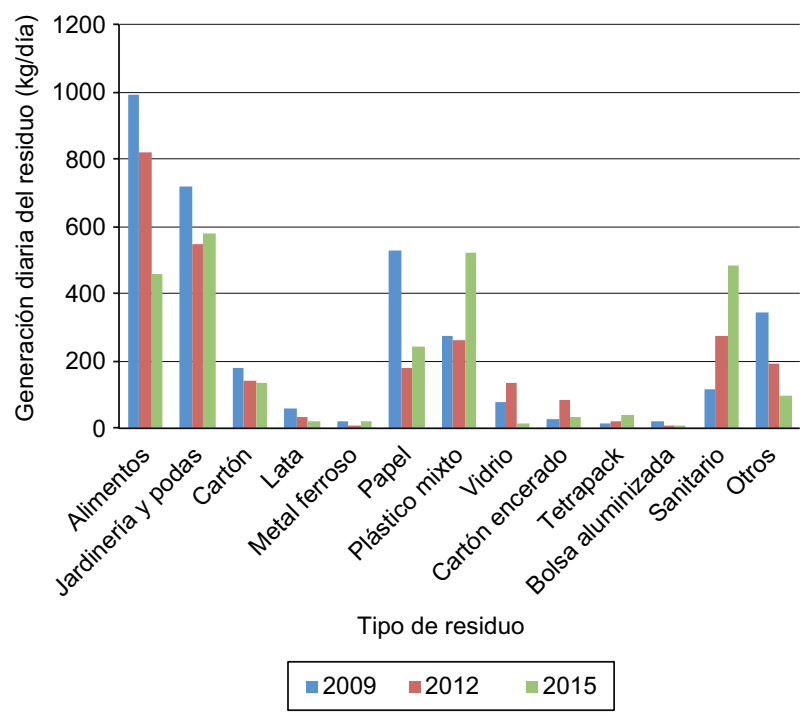

Fig. 3. Comparativo de generación diaria ( $\mathrm{kg} / \mathrm{día})$ de residuos sólidos por tipo en los años 2009, 2012 y 2015
CUADRO I. PORCENTAJE DE REDUCCIÓN EN LA GENERACIÓN DE RESIDUOS RECICLABLES EN LA UNIVERSIDAD IBEROAMERICANA CIUDAD DE MÉXICO

\begin{tabular}{lcc}
\hline Tipo de residuo & $\begin{array}{c}\text { Porcentaje de } \\
\text { reducción en la } \\
\text { generación diaria, } \\
\text { en el periodo de } \\
2009 \text { a } 2012 \\
(\%)\end{array}$ & $\begin{array}{c}\text { Porcentaje de } \\
\text { reducción en la } \\
\text { generación diaria, } \\
\text { en el periodo de } \\
2009 \text { a 2015 } \\
(\%)\end{array}$ \\
\hline Polietileno (PET) & 25.42 & 19.26 \\
Cartón & 21.94 & 19.10 \\
Aluminio & 40.58 & 73.32 \\
Papel & 65.47 & 69.50 \\
Residuos & 16.87 & 53.90 \\
Alimenticios & 23.92 & 19.53 \\
Residuos de & & \\
jardinería y podas & & \\
\hline
\end{tabular}

los años 2009, 2012 y 2015. Estos resultados se presentan de forma gráfica en la figura 4 y muestran cómo el papel, el aluminio, el PET y el Tetra Pak® presentan un aumento en el porcentaje de separación. Particularmente en el caso del papel, los resultados muestran un incremento constante en los periodos analizados.

CUADRO II. PORCENTAJE DE SEPARACIÓN DE RESIDUOS RECICLABLES EN LAUNIVERSIDAD IBEROAMERICANA CIUDAD DE MÉXICO

\begin{tabular}{lrrr}
\hline Tipo de residuo & $\begin{array}{c}2009 \\
(\%)\end{array}$ & \multicolumn{1}{c}{$\begin{array}{c}2012 \\
(\%)\end{array}$} & \multicolumn{1}{c}{$\begin{array}{c}2015 \\
(\%)\end{array}$} \\
\hline Papel & 22.93 & 31.04 & 45.97 \\
Cartón & 16.56 & 20.27 & 17.67 \\
Aluminio & 3.78 & 1.88 & 17.97 \\
Tereftalato de polietileno (PET) & 1.88 & 1.99 & 2.57 \\
Envase multicapa (Tetra Pak®) & 0.00 & 0.95 & 2.54 \\
Residuos de alimentos & 0.00 & 1.21 & 0.17 \\
Residuos de jardinería y podas & 100.00 & 100 & 92.15 \\
\hline
\end{tabular}

\section{DISCUSIÓN}

\section{Generación y composición de residuos sólidos}

Los beneficios del Plan de Manejo Integral de Residuos Sólidos se observan de manera cuantitativa en la reducción de la cantidad total de residuos generados y en el incremento de material separado para reciclaje. De manera cualitativa, también es posible observar una mejora en la actitud y participación colaborativa de los diversos miembros de la comunidad universitaria. Tanto alumnos como personal académico, administrativo y de servicio han jugado 


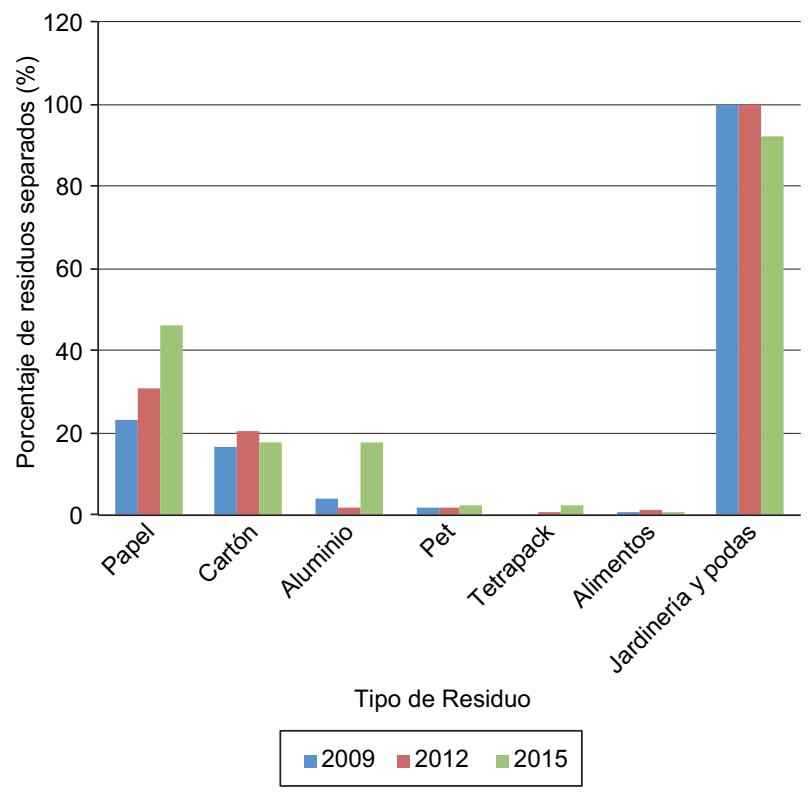

Fig. 4. Comparativo del porcentaje de separación de residuos reciclables para los años 2009, 2012 y 2015

roles determinantes para la implementación exitosa del Plan, y a partir de ello, reconocen la responsabilidad de su papel en este tema (Ruiz y Acevedo 2012).

A partir de los estudios de cuantificación y caracterización de residuos se obtuvo información detallada por tipo de residuo que puede relacionarse con las diversas acciones llevadas a cabo dentro del Plan de Manejo Integral. En materia de plásticos, aunque se reporta como una sola categoría, el estudio detallado incluye la contabilización de diversos tipos de plásticos. En particular, desde el año 2009 se prohibió a las cafeterías concesionadas el uso de empaques desechables elaborados con poliestireno expandido (unicel) para servir sus alimentos. Sin embargo, todavía se observa una cantidad considerable de unicel en la mezcla de residuos, lo cual puede atribuirse principalmente a los proveedores de las cafeterías que utilizan este material como empaque para alimentos (como en el caso de la carne que suele empacarse en charolas de unicel). Adicionalmente se encontraron diversos empaques de equipo de cómputo y consumibles electrónicos hechos de unicel. La practicidad de este material para empaque hace poco probable que la generación de los residuos de este tipo de polímero se elimine al $100 \%$ en instituciones educativas.

Por otro lado, la gran cantidad de envases multicapa (Tetra Pak®) contabilizados hace inferir que éstos no son generados en su totalidad en la Universidad sino que son transportados desde los hogares de los diversos miembros de la comunidad universitaria para dar un destino adecuado a estos residuos. Esta inferencia se soporta con diversas entrevistas orales realizadas de manera aleatoria en el período de verano y otoño de 2015 a personas que depositaban estos envases en los contenedores de residuos reciclables.

\section{Reducción}

Otro aspecto importante de hacer notar es la disminución en la generación de latas de aluminio. Se tiene notificación de la Oficina de Concesiones de la Universidad que las empresas que manejan las máquinas expendedoras reportan una disminución en el consumo de bebidas carbonatadas (refrescos) que se sustituye por el consumo de agua embotellada. Aún con esta sustitución, la generación total de botellas de PET en el campus disminuyó, ya que la política de no compra de botellas de agua para eventos y consumo en oficinas incrementó el uso del agua de garrafón proveniente de la planta potabilizadora con que cuenta la propia institución.

En el caso de la generación de residuos sanitarios, el aumento observado puede atribuirse a un incremento de la población en el campus y a las características demográficas de nuevos integrantes de la comunidad universitaria en los últimos años. La Universidad abrió sus puertas a jóvenes del nivel bachillerato en el otoño de 2010 y del nivel técnico superior universitario en la primavera de 2013, ocasionándose un incremento aproximado del $15 \%$ en la población del campus. Se podría argumentar que los adolescentes y adultos jóvenes hacen un uso mayor de las instalaciones sanitarias y generan mayor cantidad de residuos que la población madura, por lo que sería deseable un estudio adicional para poder sostener esta hipótesis.

\section{Cambios en la infraestructura del campus}

Adicionalmente, en el período analizado se tuvo un aumento importante en la infraestructura de la Universidad en ciertas áreas: se abrieron dos nuevas cafeterías (2009 y 2010) y un Centro Cultural y Deportivo (2014) en un edificio de siete niveles que incluye una cafetería adicional y múltiples baños y regaderas. Podría inferirse que este tipo de instalaciones promueven la permanencia en el campus por períodos más largos y por lo tanto una mayor generación de residuos de tipo sanitario.

En cuanto a los residuos de jardinería, aún quedan puntos a mejorar para su recolección, puesto que no se ha logrado que la totalidad de estos residuos vaya al depósito correspondiente para ser trasladados a la planta de composteo de la Delegación Álvaro 
Obregón. Una fracción de estos residuos (8 \%) va al depósito general (Cuadro II). Una posible causa de esto es la lejanía del depósito de residuos de jardinería de otras áreas ajardinadas de la Universidad, lo que hace más rápido y práctico llevar estos residuos al depósito general para que el servicio de limpia de la ciudad los retire junto con el resto de los residuos, sin garantizarse su reciclaje y composteo.

Con respecto a los residuos de alimentos, si bien se ha manifestado la conveniencia de contar con un sistema de composteo dentro del campus, el espacio para la producción y almacenaje del producto terminado, aunado a la logística existente con los concesionarios de las cafeterías, ha impedido la implementación de dicho sistema por lo que los resultados actuales del composteo in situ son limitados a la capacidad de la estación experimental de lombricomposta, que es de alrededor del $1 \%$ generado (Cuadro II).

\section{Módulos de separación de residuos reciclables}

El incremento en la separación de residuos reciclables que puede observarse en la figura 3 puede atribuirse en gran medida a la instalación de los nuevos módulos de separación de residuos reciclables, ya que durante el periodo 2013 a 2015 no se llevó a cabo ninguna campaña masiva que promoviera la separación de este tipo de residuos. De hecho, un estudio de Largo-Wight et al. (2013) concluye que, para incrementar de manera significativa la separación de residuos, lo más eficiente es colocar contenedores de manera conveniente para el usuario, independientemente de hacer o no campañas de promoción. Si bien, las campañas de comunicación y las acciones de educación ambiental masivas son un componente importante que debe prevalecer en las Instituciones de educación superior (Espinosa Valdemar et al. 2013) es también conveniente reforzar los esfuerzos para encontrar mecanismos que faciliten la gestión ambiental dentro de las propias instalaciones educativas.

En resumen, los beneficios observados de la aplicación del Plan de Manejo Integral de Residuos Sólidos fueron los siguientes:

1) Reducción de la cantidad total de residuos generados.

2) Reducción en la generación de los siguientes materiales de manera particular: latas de aluminio y botellas de PET.

3) Mayor cantidad de materiales separados para reciclaje.

4) Mayor participación colaborativa de los diversos miembros de la comunidad universitaria.

\section{CONCLUSIONES}

El Plan de Manejo Integral de Residuos Sólidos en la Universidad Iberoamericana Ciudad de México no se limita a ser una estrategia de reducción de residuos, sino que se convierte también en una herramienta educativa, al fomentar la participación colaborativa de académicos, alumnos y personal administrativo tanto en las tareas de investigación como en las de gestión en el área de manejo de residuos. La puesta en marcha de las acciones antes descritas promueve la cultura ambiental en toda la comunidad y se convierte en una herramienta para la formación de los alumnos. El impacto positivo de la gestión ambiental en la educación integral ha sido documentado por Ruiz y Acevedo (2012), quienes muestran que las diferentes actividades realizadas en la Universidad Iberoamericana Ciudad de México han propiciado que alumnos de distintos programas académicos apliquen conocimientos de áreas tan diversas como la química y la mecánica, la economía, el diseño y la planeación urbana en torno al tema de los residuos. Al mismo tiempo, se han reforzado los valores de la responsabilidad y el compromiso con el ambiente.

A través de las acciones y resultados descritos en el presente documento, la Universidad Iberoamericana Ciudad de México se propone ser un referente en cuanto al manejo integral de residuos sólidos, demostrándose que es posible reducir la cantidad de residuos generados aún a pesar del crecimiento poblacional en un campus universitario. La experiencia de los años recientes muestra que la implementación de acciones articuladas orientadas a mejorar las condiciones ambientales rinde resultados satisfactorios.

\section{AGRADECIMIENTOS}

La autora desea agradecer a las autoridades de la Universidad Iberoamericana las facilidades prestadas para la realización de la presente investigación, especialmente al personal del Programa de Medio Ambiente, así como a los alumnos que ayudaron en el estudio de campo.

\section{REFERENCIAS}

Alcántara V., Cruz I. y Santos E. (2005). Caracterización y cuantificación de los residuos sólidos en ciudad universitaria. Memorias. Congreso Interamericano de Residuos. Mérida, Yucatán, 4-7 mayo, pp. 1-88. 
Gutiérrez Barba B.E. y Martínez Rodríguez, M.C. (2010) Plan de acción sobre desarrollo sustentable en las instituciones de Educación Superior. Escenarios posibles. Revista de la Educación Superior 154, 111-132.

ARIUSA (2014) Definición de indicadores de evaluación de la sustentabilidad en Universidades Latinoamericanas. Alianza de Redes Iberoamericanas de Universidades por la Sustentabilidad y el Ambiente [en línea]. http://ariusa.net/apc-aa-fil es/66676664676466676466676466676466/201411-25-jba-version-definitiva_2.pdf 03/07/2015

Armijo C., Ojeda S. y Ramírez M.E. (2008). Solid waste characterization and recycling potential for a university campus. Waste Manag. 28 (1), S21-S26.

DOI: 10.1016/j.wasman.2008.03.022

Atherton A. y Giurco D. (2011). Campus sustainability: climate change, transport and paper reduction. Int. J. Sustain. High Educ. 12, 269-279.

DOI: $10.1108 / 14676371111148054$

Blumenstein L. (2008). Unwanted furniture finds new homes. Libr. J. 48, 38-38.

Espinosa R. M., Turpin S., Polanco G., De la Torre A., Delfín I. y Raygoza I. (2008). Integral urban solid waste management program in a Mexican university. Waste Manag. 28 (1), S27-S32.

DOI:10.1016/j.wasman.2008.03.023

Espinosa Valdemar R., Turpin Marion S., Vázquez Solís R., Vázquez Morillas A., Cisneros Ramos A., De la Torre Vega A. y García García B. (2013). La gestión ambiental en una institución de educación superior asociada a las prácticas de separación y recuperación de residuos. Rev. Int. Contam. Ambie. 29 (3), 49-57.

Fernández-Dávalos D. (2016). Mensaje del Rector. Segundo Informe de Actividades. Universidad Iberoamericana Ciudad de México [en línea]. http://www.ibero. $\mathrm{mx}$ /informedelrector 13/06/2016.

Fierro Ochoa A., Armijo de la Vega C., Buenrostro Delgado O. y Valdez Salas B. (2010). Análisis de la generación de residuos sólidos en supermercados de la Ciudad de Mexicali, México. Rev. Int. Contam. Ambie. 26 (4), 291-297.

Fournier M. (2008). Recycle this! A look at campus recycling programs. En: The green campus: Meeting the challenge of environmental sustainability (W. Simpson, Ed.). APPA, Alexandria, EUA, pp. 191-206.

Garduño Palomino K., Ojeda Benítez S. y Armijo de Vega C. (2012). Caracterización de residuos sólidos generados por el sector comercial de Mexicali, B.C. Rev. Int. Contam. Ambie. 28 (1), 19-25.

Geng Y., Liu K., Xue B. y Fujita T. (2013). Creating a "green university" in China: a case of Shenyang University. J. Clean Prod. 61, 13-19.

DOI: 10.1016/j.jclepro.2012.07.013
Goral T. (2009). E-cycle, Reboot, Reuse. Univ Bus.12, 39-39.

IBERO CAMPUS VERDE (2012). Foro de Sistemas de Manejo Ambiental [en línea]. http://ibero.mx/ campusverde/otros/foro-sistemas-manejo-ambiental/ 03/07/2015.

Largo-Wight E., Johnston D. D. y Wight J. (2013). The Efficacy of a Theory-Based, Participatory Recycling Intervention on a College Campus. J. Environ. Health 76 (4), 26-31.

Long J., Harré N. y Atkinson Q. (2014). Understanding Change in Recycling and Littering Behavior Across a School Social Network. Am. J. Commun. Psychol. 53 (3-4), 462-474. DOI: 10.1007/s10464-013-9613-3

Martín A. (2005). Compromiso Nacional por la Década de la Educación para el Desarrollo Sustentable [en línea]. http://www.anea.org.mx/docs/DEDS-CompromisoMexico.zip 10/06/2016.

Nieto-Caraveo L.M. (2001) El Consorcio Mexicano de Programas Ambientales Universitarios para el Desarrollo Sustentable: Enfoque y Desafíos. Revista Universitarios 8 (6), 1-10.

Posey M. J. y Webster A. H. (2013). Environmental Sustainability Practices in Publicly Supported Two-Year Colleges in the Southern United States. Community College Journal Of Research and Practice 37 (10), 800-803. DOI: $10.1080 / 10668926.2012 .763148$

Ruiz Morales M. (2012). Caracterización de residuos sólidos en la Universidad Iberoamericana Ciudad de México. Rev. Int. Contam. Ambie. 28 (1), 93-97.

Ruiz M. y Acevedo A. (2012). Inclusion of Environmental Education in Various Engineering Courses through an Integrated Solid Waste Management Program. En: Innovations 2012: World Innovations in Engineering Education and Research (W. Aung, J. Moscinski, M.G. Rasteiro, I. Rouse, B. Wagner y P. Willmot, Eds.). Begell House Publishing, Arlington, EUA, pp. 217-226.

Saldaña Durán C., Hernández Rosales I., Messina Fernández S. y Pérez Pimienta J. (2013). Caracterización de los residuos sólidos urbanos y el valor agregado de los materiales recuperables en el vertedero El Iztete, de Tepic-Nayarit, México. Rev. Int. Contam. Ambie. 29 (3), 25-32.

SECOFI (1985a). Norma Mexicana NMX-AA-015-1985. Protección al Ambiente-Contaminación del SueloResiduos Sólidos Municipales-Muestreo-Método de Cuarteo. Secretaría de Comercio y Fomento Industrial. Diario Oficial de la Federación. 18 marzo de 1985.

SECOFI (1985b). Norma Mexicana NMX-AA-022-1985. Protección al Ambiente-Contaminación del SueloResiduos Sólidos Municipales-Selección y Cuantificación de Subproductos. Secretaría de Comercio y Fomento Industrial. Diario Oficial de la Federación. 18 marzo de 1985. 
SECOFI (1985c). Norma Mexicana NMX-AA-61-1985. Protección al Ambiente-Contaminación del Suelo-Residuos Sólidos Municipales-Determinación de la Generación. Secretaría de Comercio y Fomento Industrial. Diario Oficial de la Federación. 8 de agosto de 1985.
Taboada-González P., Aguilar-Virgen Q., Cruz-Sotelo S. y Ramírez-Barreto M. (2013). Manejo y potencial de recuperación de residuos sólidos en una comunidad rural de México. Rev. Int. Contam. Ambie. 29 (3), 43-48. 